\title{
Familial or Sporadic Idiopathic Scoliosis - classification based on artificial neural network and GAPDH and ACTB transcription profile
}

\author{
Tomasz Waller $^{1 *+}$, Roman Nowak ${ }^{2 \dagger}$, Magdalena Tkacz ${ }^{4 \dagger}$, Damian Zapart ${ }^{1 \dagger}$ and Urszula Mazurek ${ }^{3+}$
}

\author{
* Correspondence: twaller@biobyte.pl \\ ${ }^{\dagger}$ Equal contributors \\ ${ }^{1}$ Institute of Computer Science, \\ Division of Biomedical Computer \\ Systems, University of Silesia, \\ Katowice, Poland \\ Full list of author information is \\ available at the end of the article
}

\begin{abstract}
Background: Importance of hereditary factors in the etiology of Idiopathic Scoliosis is widely accepted. In clinical practice some of the IS patients present with positive familial history of the deformity and some do not. Traditionally about $90 \%$ of patients have been considered as sporadic cases without familial recurrence. However the exact proportion of Familial and Sporadic Idiopathic Scoliosis is still unknown. Housekeeping genes encode proteins that are usually essential for the maintenance of basic cellular functions. ACTB and GAPDH are two housekeeping genes encoding respectively a cytoskeletal protein $\beta$-actin, and glyceraldehyde-3-phosphate dehydrogenase, an enzyme of glycolysis. Although their expression levels can fluctuate between different tissues and persons, human housekeeping genes seem to exhibit a preserved tissue-wide expression ranking order. It was hypothesized that expression ranking order of two representative housekeeping genes ACTB and GAPDH might be disturbed in the tissues of patients with Familial Idiopathic Scoliosis (with positive family history of idiopathic scoliosis) opposed to the patients with no family members affected (Sporadic Idiopathic Scoliosis). An artificial neural network (ANN) was developed that could serve to differentiate between familial and sporadic cases of idiopathic scoliosis based on the expression levels of ACTB and GAPDH in different tissues of scoliotic patients. The aim of the study was to investigate whether the expression levels of ACTB and GAPDH in different tissues of idiopathic scoliosis patients could be used as a source of data for specially developed artificial neural network in order to predict the positive family history of index patient.
\end{abstract}

Results: The comparison of developed models showed, that the most satisfactory classification accuracy was achieved for ANN model with 18 nodes in the first hidden layer and 16 nodes in the second hidden layer. The classification accuracy for positive Idiopathic Scoliosis anamnesis only with the expression measurements of ACTB and GAPDH with the use of ANN based on 6-18-16-1 architecture was 8 of 9 (88\%). Only in one case the prediction was ambiguous.

Conclusions: Specially designed artificial neural network model proved possible association between expression level of ACTB, GAPDH and positive familial history of Idiopathic Scoliosis.

\section{Biomed Central}

(c) 2013 Waller et al.; licensee BioMed Central Ltd. This is an Open Access article distributed under the terms of the Creative Commons Attribution License (http://creativecommons.org/licenses/by/2.0), which permits unrestricted use, distribution, and reproduction in any medium, provided the original work is properly cited. 


\section{Background}

Idiopathic Scoliosis (IS) is the most common structural deformity of the human spine. Although the exact cause or causes of idiopathic scoliosis are still unknown there is convincing evidence supporting a genetic aetiology of this disorder [1-5]. Importance of hereditary factors in the etiology of IS is underlined by increased risk of scoliosis among the first-degree relatives of index patients. Harrington found scoliosis incidence of $27 \%$ among the first degree relatives. [6] Other studies indicate $11 \%$ of first degree and $2,4 \%$ and $1,4 \%$ of second and third degree relatives are affected [7,8]. Genetic basis of IS is also supported by the results of the twin studies. Inoue and colleagues found the concordance rate of scoliosis of $92,3 \%$ in monozygous, decreasing to $62,5 \%$ in dizygous twins [9]. Lower concordance rate was found in the study of Kesling and al, 73\% among monozygous and 36\% among dizygous twins [10]. Recent study based on the Swedish Twin Registry estimates that heritability of this condition is $38 \%$ indicating the importance of other still unknown factors in the development of the deformity [11]. Mode of inheritance and genetic basis of the scoliotic phenotype are still not definitively determined. Autosomal dominant mode, X-linked as well as multifactorial inheritance patterns were suggested [3-7]. According to Miller et al. idiopathic scoliosis is a complex genetic disorder involving one or more genetic loci and complex genetic interactions for disease expression [5]. In clinical practice some of the IS patients present with positive familial history of the deformity and some do not. Traditionally about 90\% of patients have been considered as sporadic cases without familial recurrence [1]. However the exact proportion of Familial and Sporadic Idiopathic Scoliosis is still unknown [5]. Ogilvie et al. in the population study based on a large data base from Utah conclude that $97 \%$ of their patients with idiopathic scoliosis have familial origins and suggest a presence of one or more major gene defects or single gene defects influenced by other factors [11]. According to Cheng et al. predisposition for IS doesn't have a specific assigned risk of heritability, but inheritance is based on multiple factors potentially both genetic and environmental, which have yet to be defined [1].

Housekeeping genes encode proteins that are usually essential for the maintenance of basic cellular functions. They are expressed constitutively in every human cell but it appears that their transcriptional level may be influenced by numerous factors [12,13]. ACTB and GAPDH are two housekeeping genes encoding respectively a cytoskeletal protein $\beta$-actin, and glyceraldehyde-3-phosphate dehydrogenase, an enzyme of glycolysis [12]. Based on the assumption of their constant expression in various tissues these genes serve as traditional internal control in a variety of assays in molecular biology [13]. Although their expression levels can fluctuate between different tissues and persons, human housekeeping genes seem to exhibit a preserved tissue-wide expression ranking order [14].

It was hypothesized that expression ranking order of two representative housekeeping genes ACTB and GAPDH might be disturbed in the tissues of patients with Familial Idiopathic Scoliosis (with positive family history of idiopathic scoliosis) opposed to the patients with no family members affected (Sporadic Idiopathic Scoliosis). In order to recognize potentially sophisticated patterns in the data and because of the tensor structure of the ACTB and GAPDH expression an artificial neural network (ANN) was developed that could serve to differentiate between familial and sporadic cases of idiopathic scoliosis based on the expression levels of ACTB and GAPDH in different tissues of scoliotic patients. 
The aim of the study was to investigate whether the expression levels of ACTB and GAPDH in different tissues of idiopathic scoliosis patients could be used as source of data for specially developed artificial neural network in order to predict the positive family history of index patients.

\section{Methods}

Patients

Study design was approved by Bioethical Committee Board of Silesian Medical University. Informed, written consent was obtained from each patient participating in the study and if required from their parents. Twenty nine patients (23 females and 6 males) with a definite diagnosis of late onset Idiopathic Scoliosis were included in the study. Thirteen of them (44\%) had positive familial history of IS. All of the patients had undergone posterior corrective surgery with segmental spinal instrumentation according to C-D method. The mean age at surgery was 16 years 8 months (range 13,7 - 24 years). Based on Lenke classification 6 curves were of type 1,6 curves of type 2,7 curves of type 3,7 curves of type 4,4 curves of type 5 and 3 of type 6 [15]. Preoperatively the average frontal and sagittal Cobb angles measured on standard p-a and lateral radiograms were $68,8^{\circ}\left(\right.$ range $36^{\circ}-114^{\circ}$ ) and $35,4^{\circ}$ (range $12^{\circ}-70^{\circ}$ ) respectively. The axial plane deformity was measured on CT scans performed at the curve apex by spinal rotation angle relative to sagittal plane RAsag and rib hump index RHi as described by Aaro and Dahlborn [16]. The mean RAsag was 19,3 (range 2,5 $-46^{\circ}$ ) and RHi 0,4 (range 0,03-0,91). During surgery bilateral facet removal was performed in the routine manner and bone specimens from inferior articular spinal processes at the curve apex concavity and convexity were harvested. In the same time bilateral samples of paravertebral muscle tissue at the apical level and $10 \mathrm{ml}$ of patients peripheral blood were collected. Every sample of bone and muscular tissue as well as blood specimens were placed in separate sterile tubes, adequately identified and immediately snap frozen in liquid nitrogen and stored at $-80^{\circ} \mathrm{C}$ until molecular analysis.

\section{Laboratory procedures}

Tissues samples were homogenized with the use of Polytron ${ }^{\circledR}$ (Kinematyka AG). Total RNA was isolated from tissue samples with the use of TRIZOL ${ }^{\circledR}$ reagent (Invitrogen Life Technologies, California, USA), according to the manufacturer's instructions. Extracts of total RNA were treated with DNAase I (Qiagen Gmbh, Hilden, Germany) and purified with the use of RNeasy Mini Spin Kolumn (Qiagen Gmbh, Hilden, Germany), in accordance with manufacturer's protocol. The quality of RNA was estimated by electrophoresis on a $1 \%$ agarose gel stained with ethidium bromide. The RNA concentration was determined by absorbance at $260 \mathrm{~nm}$ using a Gene Quant II spectrophotometer (Pharmacia LKB Biochrom Ltd., Cambridge, UK). Total RNA served as a matrix for QRT PCR.

ACTB and GAPDH mRNA quantification in osseous, muscular and blood tissue samples by Quantitative Real Time Reverse Transcription Polymerase Chain Reaction.

The quantitative analysis was carried out with the use of Sequence Detector ABI PRISM $^{\mathrm{TM}} 7000$ (Applied Biosystems, California, USA). The standard curve was appointed for standards of ACTB (TaqMan ${ }^{\circledR}$ DNA Template Reagents Kit, Applied 
Biosystems, Foster, CA, USA). The ACTB and GAPDH mRNA abundance in all studied tissue specimens were expressed as mRNA copy number per $1 \mu \mathrm{g}$ of total RNA.

The QRT-PCR reaction mixture of a total volume of $25 \mu \mathrm{l}$ contained QuantiTect SYBR- Green RT-PCR bufor containing Tris-HCl $\left(\mathrm{NH}_{4}\right)_{2} \mathrm{SO}_{4}, 5 \mathrm{mM} \mathrm{MgCl} 2, \mathrm{pH} 8,7$, dNTP mix fluorescent dye SYBR-Green I, and passive reference dye ROX mixed with 0,5 $\mu$ l QuantiTect RT mix (Omniscript reverse transcriptase, Sensiscript reverse transcriptase) (QuantiTect SYBR-Green RT-PCR kit; Qiagen) forward and reverse primers each at a final concentration of $0,5 \mu \mathrm{M}$ mRNA and total RNA $0,25 \mu \mathrm{g}$ per reaction. Sequence for primers: mRNA for mRNA for ACTB 5'TCACCCACACTGTGCCC ATC TACGA3'(forward primer) 5'CAGCGGAACCGCTCATTGCCAATGG3' (reverse primer), mRNA for GAPDH 5'GAAGGTGAAGGTCGGAGTC3'(forward primer) 5'GAA GATGG TGATGGGATT 3'(reverse primer). Reverse transcription was carried out at $50^{\circ} \mathrm{C}$ for $30 \mathrm{~min}$. After activation of the HotStar Taq DNA polymerase and deactivation of reverse transcriptases at $95^{\circ} \mathrm{C}$ for $15 \mathrm{~min}$, subsequent PCR amplification consisted of denaturation at $94^{\circ} \mathrm{C}$ for $15 \mathrm{sec}$, annealing at $60^{\circ} \mathrm{C}$ for $30 \mathrm{sec}$ and extension at $72^{\circ} \mathrm{C}$ for $30 \mathrm{sec}$ ( 40 cycles). Final extension was carried out at $72^{\circ} \mathrm{C}$ for $10 \mathrm{~min}$. QRT-PCR specificity was assessed by electrophoresis in $6 \%$ polyacrylamid gel and melting curves for aplimeres.

\section{Patient data}

The results of laboratory procedures and the family anamnesis of 29 patients were used to create dataset consisting of 29 rows. The expression values were transformed to

Table 1 Training set

\begin{tabular}{cccccccc}
\hline ID & $\begin{array}{c}\text { GAPDH } \\
\text { bone } \\
\text { concavity) }\end{array}$ & $\begin{array}{c}\text { ACTB bone } \\
\text { (concavity) }\end{array}$ & $\begin{array}{c}\text { GAPDH } \\
\text { muscle } \\
\text { (concavity) }\end{array}$ & $\begin{array}{c}\text { ACTB } \\
\text { muscle } \\
\text { (concavity) }\end{array}$ & $\begin{array}{c}\text { GAPDH } \\
\text { blood }\end{array}$ & ACTB blood & CLASSIFICATION \\
\hline 1 & 0 & 3,655234507 & 5,19447549 & 5,492043421 & $\mathbf{0 , 7 8 8 0 3 6 6 1 5}$ & $\mathbf{7 , 9 4 7 0 5 5 4 3 2}$ & 1 - Familial IS \\
\hline 2 & 2,260071388 & 2,681241237 & 5,492043421 & 5,19447549 & 4,351834943 & 4,379378045 & 0 \\
\hline 3 & 1,361727836 & 0 & $\mathbf{6 , 8 8 3 0 5 4 4 5 9}$ & $\mathbf{1 , 9 3 2 9 4 6 8 1 6}$ & 3,543198586 & 7,878946654 & 0 \\
\hline 4 & 2,26245109 & 4,490393961 & 5,010236335 & 5,166876908 & 4,484314078 & 4,062506775 & 1 \\
\hline 5 & 0,77815125 & 4,52146499 & $\mathbf{5 , 2 3 9 9 6 6 2 9 6}$ & $\mathbf{4 , 4 3 5 9 3 7 3 1 3}$ & 1,838849091 & 5,133344071 & 1 \\
\hline 6 & 0 & 4,15192118 & 5,160648574 & 4,706444663 & 2,46686762 & 4,210666244 & 1 \\
\hline 7 & 0 & 1,342422681 & 2,822821645 & 3,140193679 & 2,041392685 & 3,7294887769 & 0 \\
\hline 8 & 0 & 1,740362689 & $\mathbf{8 , 4 1 6 7 7 3 1 8 7}$ & $\mathbf{1 , 3 0 7 7 3 7 9 0 2}$ & 1,886490725 & 3,820595497 & 0 \\
\hline 9 & 2,7084209 & 3,68797462 & $\mathbf{7 , 9 0 6 9 4 8 8 5 5}$ & $\mathbf{4 , 8 4 2 9 3 9 9 0 8}$ & 2,283301229 & 3,062205809 & 1 \\
\hline 10 & 1,77815125 & 3,496376054 & 2,559906625 & 3,126131407 & 4,614992076 & 4,872779577 & 0 \\
\hline 11 & 0 & 6,053585081 & 0 & 4,704202011 & $\mathbf{5 , 7 5 3 3 7 6 8 3 8}$ & $\mathbf{0 , 8 8 7 4 2 5 8}$ & 0 \\
\hline 12 & 0 & 3,331022171 & 4,2175629 & 4,527707216 & 5,322554193 & 5,961483267 & 0 \\
\hline 13 & 1,204119983 & 4,060168812 & 3,882068944 & 4,669075022 & 5,979840083 & 4,431492425 & 0 \\
\hline 14 & 10,59979533 & 0 & 3,257198426 & 4,146065989 & 3,834102656 & 3,931152639 & 1 \\
\hline 15 & 0 & 1,146128036 & 0,903089987 & 3,761401557 & 2,380211242 & 5,055026472 & 1 \\
\hline 16 & 0 & 2,765668555 & 0 & 3,709015417 & 3,087426457 & 4,830563008 & 0 \\
\hline 17 & 0 & 1,792391689 & 0 & 4,713734083 & 3,098643726 & 5,036968055 & 0 \\
\hline 18 & 4,443841661 & 2,615950052 & 1,505149978 & 4,414388327 & 3,128076013 & 4,702835345 & 1 \\
\hline 19 & 7,280817804 & 2,555094449 & 0 & 2,029383778 & 0 & 2,271841607 & 0 \\
\hline 20 & 0 & 0,301029996 & 1,944482672 & 4,584489532 & 3,036628895 & 4,451325808 & 1 \\
\hline The random values are marked in bold. & & & & & \\
\hline
\end{tabular}


Table 2 Test set and ANN's prediction

\begin{tabular}{|c|c|c|c|c|c|c|c|c|c|c|}
\hline ID & $\begin{array}{c}\text { GAPDH } \\
\text { bone } \\
\text { (concavity) }\end{array}$ & $\begin{array}{l}\text { ACTB bone } \\
\text { (concavity) }\end{array}$ & $\begin{array}{c}\text { GAPDH } \\
\text { muscle e } \\
\text { (concavity) }\end{array}$ & $\begin{array}{c}\text { ACTB } \\
\text { muscle } \\
\text { (concavity) }\end{array}$ & $\begin{array}{l}\text { GAPDH } \\
\text { blood }\end{array}$ & $\begin{array}{l}\text { ACTB } \\
\text { blood }\end{array}$ & $\frac{\frac{\text { CLASSIFICATION }}{0-\text { Sporadic IS }}}{1-\text { Familial IS }}$ & $\begin{array}{l}6-18-16-1 \\
\text { ANN'S } \\
\text { PREDICTION }\end{array}$ & $\begin{array}{l}6-19-19-1 \\
\text { ANN'S } \\
\text { PREDICTION }\end{array}$ & $\begin{array}{l}6-18-10-1 \\
\text { ANN'S } \\
\text { PREDICTION }\end{array}$ \\
\hline 21 & 0 & 2,999130541 & 2,086359831 & 4,54961624 & 2,595496222 & 5,315582034 & 1 & 0,8150237 & 0,0658464 & 0,9729949 \\
\hline 22 & 0 & 3,325104983 & 2,981365509 & 3,940018155 & 3,253822439 & 5,188225173 & 0 & 0,1371327 & 0,0111759 & 0,9755903 \\
\hline 23 & 4,259641653 & 4,832872801 & 6,266957346 & 4,19709909 & 4,228759555 & 4,237141427 & 1 & 0,9970080 & 0,8874323 & 0,9444540 \\
\hline 24 & 1,041392685 & 3,29136885 & 6,118608586 & 5,357498429 & 1,036299441 & 3,74587204 & 1 & 0,9984768 & 0,9984370 & 0,9981375 \\
\hline 25 & 0,84509804 & 4,850768727 & 1,176091259 & 3,542451947 & 2,305351369 & 3,743744879 & 0 & 0,0203165 & 0,0046074 & 0,0195937 \\
\hline 26 & 2,598790507 & 4,308116016 & 5,95395578 & 5,604965452 & 3,836324116 & 3,596047008 & 1 & 0,9979908 & 0,9941236 & 0,9964907 \\
\hline 27 & 0 & 3,804275767 & 5,556972498 & 3,33701319 & 3,055760465 & 3,388811413 & 0 & 0,3232977 & 0,2974088 & 0,0672966 \\
\hline 28 & 0 & 3,513483957 & 0,805059074 & 2,195759967 & 1,176091259 & 3,688508808 & 0 & 0,0371065 & 0,0023705 & 0,0179424 \\
\hline 29 & 0 & 4,132643851 & 0,84509804 & 4,909245708 & 1,579783597 & 4,111497749 & 0 & 0,0338294 & 0,0341857 & 0,0104404 \\
\hline
\end{tabular}


logarithmic scale. One row represented ACTB and GAPDH transcription profile in three kinds of tissue (bone, muscle, and blood) for exactly one patient.

Unfortunately, there were some missing data in our dataset. To face this problem we could either remove incomplete records from the analyzed dataset or use appropriate methodology and tool to preserve and utilize them in the analysis. In data mining and knowledge discovery from data disciplines the problem of missing data is widely discussed [17-22]. With the removal of all incomplete records we could risk losing some important information contained in the whole dataset. In effect we decided to preserve all the records and replace missing values by random data from normal distributions similar to the original distributions of the variables. The random values were marked in bold [Tables 1 and 2]. Our decision was supported by the experience of one of the coauthors of this study conducting extensive research in the field of advanced data processing therein in processing incomplete data [23-27]. Basing on the mentioned above ANN was chosen as an appropriate method for classification in this case. The dataset was randomly divided into training set (20 rows) [Table 1 ] and test set (9 rows) [Table 2].

\section{Artificial neural network}

Artificial neural network is a mathematical model that is inspired by the structure and functional aspects of biological neural networks [28,29]. ANN can be used to detect sophisticated patterns in data. Several studies have applied neural networks in research and analysis of various diseases (i.e. classification of cardiovascular disease, forecast for bacteria - antibiotic interactions, prediction of colorectal cancer patient survival) [30].

The architecture of the ANN used in this study is the multilayered feed-forward network architecture with four layers (two hidden layers). Multilayer feed-forward neural networks can be used to approximate a nonlinear functions which are applied to describe the complicated relationships in biological data [31]. The schematic

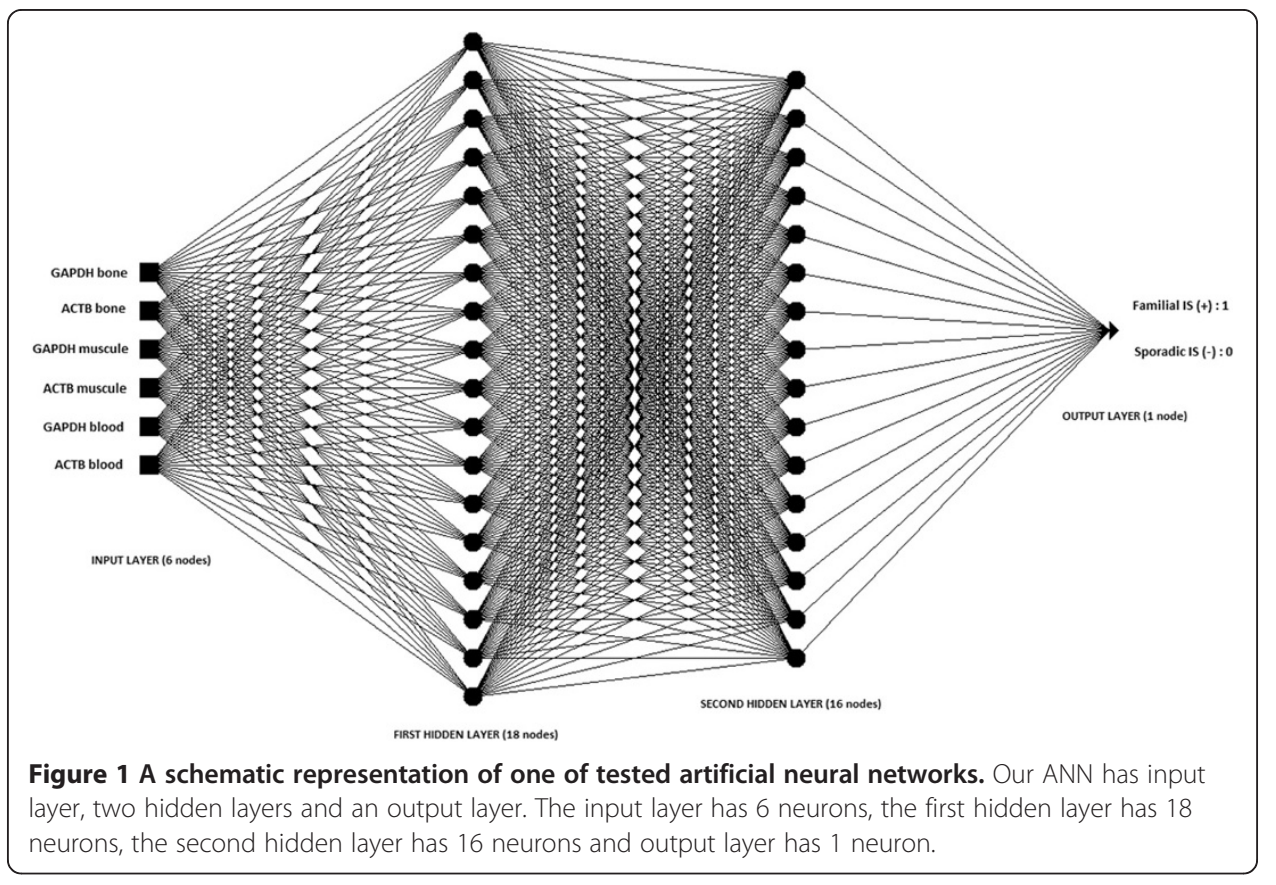


Table 3 Evaluation and selection of multiple network architectures

\begin{tabular}{|c|c|c|c|c|c|c|c|c|c|c|c|c|c|c|c|c|c|c|c|}
\hline No. & $\begin{array}{c}\text { ANN } \\
\text { architecture }\end{array}$ & $\begin{array}{c}\text { MSE } \\
50 \\
\text { epochs }\end{array}$ & $\begin{array}{l}\text { MSE } \\
500 \\
\text { epochs }\end{array}$ & $\begin{array}{l}\text { MSE } \\
5000 \\
\text { epochs }\end{array}$ & No. & $\begin{array}{c}\text { ANN } \\
\text { architecture }\end{array}$ & $\begin{array}{c}\text { MSE } \\
50 \\
\text { epochs }\end{array}$ & $\begin{array}{c}\text { MSE } \\
500 \\
\text { epochs }\end{array}$ & $\begin{array}{l}\text { MSE } \\
5000 \\
\text { epochs }\end{array}$ & No. & $\begin{array}{c}\text { ANN } \\
\text { architecture }\end{array}$ & $\begin{array}{c}\text { MSE } \\
50 \\
\text { epochs }\end{array}$ & $\begin{array}{l}\text { MSE } \\
500 \\
\text { epochs }\end{array}$ & $\begin{array}{c}\text { MSE } \\
5000 \\
\text { epochs }\end{array}$ & No. & $\begin{array}{c}\text { ANN } \\
\text { architecture }\end{array}$ & $\begin{array}{c}\text { MSE } \\
50 \\
\text { epochs }\end{array}$ & $\begin{array}{c}\text { MSE } \\
500 \\
\text { epochs }\end{array}$ & $\begin{array}{c}\text { MSE } \\
5000 \\
\text { epochs }\end{array}$ \\
\hline 1 & $6-18-16-1$ & 0,426 & 0,058 & 0,006 & 57 & $6-16-8-1$ & 0,355 & 0,048 & 0,008 & 113 & $6-12-7-1$ & 0,432 & 0,059 & 0,009 & 169 & $6-5-11-1$ & 0,490 & 0,308 & 0,011 \\
\hline 2 & $6-19-19-1$ & 0,413 & 0,049 & 0,006 & 58 & $6-16-11-1$ & 0,328 & 0,046 & 0,008 & 114 & $6-11-1-1$ & 0,384 & 0,069 & 0,009 & 170 & $6-8-9-1$ & 0,483 & 0,057 & 0,011 \\
\hline 3 & $6-18-10-1$ & 0,440 & 0,045 & 0,006 & 59 & $6-19-15-1$ & 0,443 & 0,052 & 0,008 & 115 & $6-16-3-1$ & 0,426 & 0,087 & 0,009 & 171 & $6-4-17-1$ & 0,497 & 0,299 & 0,011 \\
\hline 4 & $6-20-11-1$ & 0,382 & 0,038 & 0,007 & 60 & $6-15-1-1$ & 0,439 & 0,121 & 0,008 & 116 & $6-10-10-1$ & 0,454 & 0,104 & 0,009 & 172 & $6-6-16-1$ & 0,445 & 0,218 & 0,011 \\
\hline 5 & $6-18-5-1$ & 0,391 & 0,047 & 0,007 & 61 & $6-18-3-1$ & 0,444 & 0,043 & 0,008 & 117 & $6-11-16-1$ & 0,408 & 0,065 & 0,009 & 173 & $6-6-15-1$ & 0,495 & 0,071 & 0,011 \\
\hline 6 & $6-19-11-1$ & 0,436 & 0,041 & 0,007 & 62 & $6-17-2-1$ & 0,417 & 0,045 & 0,008 & 118 & $6-8-20-1$ & 0,420 & 0,194 & 0,009 & 174 & $6-6-7-1$ & 0,463 & 0,172 & 0,011 \\
\hline 7 & $6-19-18-1$ & 0,438 & 0,042 & 0,007 & 63 & $6-15-14-1$ & 0,420 & 0,066 & 0,008 & 119 & $6-14-3-1$ & 0,477 & 0,041 & 0,009 & 175 & $6-5-20-1$ & 0,485 & 0,106 & 0,011 \\
\hline 8 & $6-20-13-1$ & 0,433 & 0,051 & 0,007 & 64 & $6-11-4-1$ & 0,352 & 0,053 & 0,008 & 120 & $6-9-16-1$ & 0,482 & 0,112 & 0,009 & 176 & $6-9-7-1$ & 0,402 & 0,065 & 0,011 \\
\hline 9 & $6-17-9-1$ & 0,416 & 0,060 & 0,007 & 65 & $6-14-18-1$ & 0,398 & 0,141 & 0,008 & 121 & $6-15-3-1$ & 0,400 & 0,049 & 0,009 & 177 & $6-8-15-1$ & 0,429 & 0,121 & 0,011 \\
\hline 10 & $6-19-14-1$ & 0,439 & 0,085 & 0,007 & 66 & $6-9-8-1$ & 0,482 & 0,059 & 0,008 & 122 & $6-9-2-1$ & 0,396 & 0,065 & 0,009 & 178 & $6-8-5-1$ & 0,396 & 0,054 & 0,012 \\
\hline 11 & $6-20-19-1$ & 0,412 & 0,042 & 0,007 & 67 & $6-15-2-1$ & 0,329 & 0,045 & 0,008 & 123 & $6-11-17-1$ & 0,398 & 0,051 & 0,009 & 179 & $6-5-17-1$ & 0,429 & 0,225 & 0,012 \\
\hline 12 & $6-18-19-1$ & 0,422 & 0,063 & 0,007 & 68 & $6-20-17-1$ & 0,352 & 0,044 & 0,008 & 124 & $6-7-20-1$ & 0,435 & 0,069 & 0,009 & 180 & $6-5-8-1$ & 0,437 & 0,312 & 0,012 \\
\hline 13 & $6-17-4-1$ & 0,417 & 0,091 & 0,007 & 69 & $6-9-14-1$ & 0,490 & 0,060 & 0,008 & 125 & $6-8-18-1$ & 0,460 & 0,187 & 0,009 & 181 & $6-5-15-1$ & 0,370 & 0,215 & 0,012 \\
\hline 14 & $6-20-8-1$ & 0,392 & 0,046 & 0,007 & 70 & $6-14-9-1$ & 0,367 & 0,049 & 0,008 & 126 & $6-10-1-1$ & 0,340 & 0,081 & 0,009 & 182 & $6-4-20-1$ & 0,491 & 0,178 & 0,012 \\
\hline 15 & $6-18-7-1$ & 0,390 & 0,079 & 0,007 & 71 & $6-12-12-1$ & 0,424 & 0,067 & 0,008 & 127 & $6-12-16-1$ & 0,438 & 0,045 & 0,009 & 183 & $6-7-3-1$ & 0,410 & 0,147 & 0,012 \\
\hline 16 & $6-14-14-1$ & 0,423 & 0,085 & 0,007 & 72 & $6-16-5-1$ & 0,388 & 0,070 & 0,008 & 128 & $6-10-17-1$ & 0,425 & 0,138 & 0,009 & 184 & $6-4-13-1$ & 0,453 & 0,269 & 0,012 \\
\hline 17 & $6-18-8-1$ & 0,363 & 0,055 & 0,007 & 73 & $6-13-5-1$ & 0,450 & 0,071 & 0,008 & 129 & $6-7-1-1$ & 0,430 & 0,084 & 0,009 & 185 & $6-6-18-1$ & 0,490 & 0,221 & 0,012 \\
\hline 18 & $6-19-4-1$ & 0,365 & 0,045 & 0,007 & 74 & $6-8-14-1$ & 0,445 & 0,076 & 0,008 & 130 & $6-10-14-1$ & 0,414 & 0,112 & 0,009 & 186 & $6-4-4-1$ & 0,446 & 0,483 & 0,012 \\
\hline 19 & $6-20-7-1$ & 0,440 & 0,087 & 0,007 & 75 & $6-17-18-1$ & 0,432 & 0,095 & 0,008 & 131 & $6-9-15-1$ & 0,442 & 0,149 & 0,009 & 187 & $6-3-12-1$ & 0,491 & 0,216 & 0,012 \\
\hline 20 & $6-20-6-1$ & 0,451 & 0,044 & 0,007 & 76 & $6-8-8-1$ & 0,486 & 0,254 & 0,008 & 132 & $6-16-16-1$ & 0,430 & 0,093 & 0,009 & 188 & $6-3-8-1$ & 0,465 & 0,102 & 0,013 \\
\hline 21 & $6-15-13-1$ & 0,431 & 0,087 & 0,007 & 77 & $6-13-4-1$ & 0,423 & 0,071 & 0,008 & 133 & $6-11-19-1$ & 0,467 & 0,107 & 0,009 & 189 & $6-6-3-1$ & 0,469 & 0,066 & 0,013 \\
\hline 22 & $6-18-6-1$ & 0,357 & 0,046 & 0,007 & 78 & $6-14-8-1$ & 0,439 & 0,064 & 0,008 & 134 & $6-13-16-1$ & 0,459 & 0,084 & 0,010 & 190 & $6-12-5-1$ & 0,373 & 0,052 & 0,013 \\
\hline 23 & $6-16-13-1$ & 0,467 & 0,045 & 0,007 & 79 & $6-20-10-1$ & 0,410 & 0,043 & 0,008 & 135 & $6-8-10-1$ & 0,404 & 0,053 & 0,010 & 191 & $6-4-1-1$ & 0,486 & 0,114 & 0,013 \\
\hline 24 & $6-13-17-1$ & 0,463 & 0,069 & 0,007 & 80 & $6-12-4-1$ & 0,439 & 0,081 & 0,008 & 136 & $6-9-3-1$ & 0,474 & 0,145 & 0,010 & 192 & $6-3-20-1$ & 0,476 & 0,353 & 0,013 \\
\hline
\end{tabular}


Table 3 Evaluation and selection of multiple network architectures (Continued)

\begin{tabular}{|c|c|c|c|c|c|c|c|c|c|c|c|c|c|c|c|c|c|c|c|}
\hline 25 & $6-17-17-1$ & 0,425 & 0,045 & 0,008 & 81 & $6-15-20-1$ & 0,444 & 0,096 & 0,008 & 137 & $6-18-1-1$ & 0,337 & 0,083 & 0,010 & 193 & $6-6-20-1$ & 0,468 & 0,059 & 0,013 \\
\hline 26 & $6-16-6-1$ & 0,413 & 0,053 & 0,008 & 82 & $6-17-20-1$ & 0,421 & 0,058 & 0,008 & 138 & $6-6-1-1$ & 0,477 & 0,335 & 0,010 & 194 & $6-3-2-1$ & 0,474 & 0,158 & 0,013 \\
\hline 27 & $6-19-9-1$ & 0,422 & 0,174 & 0,008 & 83 & $6-13-11-1$ & 0,395 & 0,094 & 0,008 & 139 & $6-8-3-1$ & 0,390 & 0,066 & 0,010 & 195 & $6-3-6-1$ & 0,498 & 0,180 & $0] 014$ \\
\hline 28 & $6-13-8-1$ & 0,419 & 0,048 & 0,008 & 84 & $6-17-6-1$ & 0,428 & 0,054 & 0,008 & 140 & $6-8-16-1$ & 0,396 & 0,127 & 0,010 & 196 & $6-20-1$ & 0,327 & 0,066 & 0,014 \\
\hline 29 & $6-13-18-1$ & 0,391 & 0,054 & 0,008 & 85 & $6-15-12-1$ & 0,432 & 0,043 & 0,008 & 141 & $6-10-12-1$ & 0,424 & 0,124 & 0,010 & 197 & $6-4-10-1$ & 0,485 & 0,177 & 0,014 \\
\hline 30 & $6-16-10-1$ & 0,383 & 0,052 & 0,008 & 86 & $6-14-2-1$ & 0,391 & 0,132 & 0,008 & 142 & $6-10-6-1$ & 0,461 & 0,073 & 0,010 & 198 & $6-8-19-1$ & 0,452 & 0,099 & 0,014 \\
\hline 31 & $6-13-20-1$ & 0,449 & 0,112 & 0,008 & 87 & $6-12-1-1$ & 0,448 & 0,044 & 0,008 & 143 & $6-6-4-1$ & 0,418 & 0,068 & 0,010 & 199 & $6-15-1$ & 0,323 & 0,073 & 0,014 \\
\hline 32 & $6-12-11-1$ & 0,454 & 0,116 & 0,008 & 88 & $6-17-8-1$ & 0,361 & 0,044 & 0,008 & 144 & $6-5-14-1$ & 0,478 & 0,207 & 0,010 & 200 & $6-15-15-1$ & 0,472 & 0,045 & 0,015 \\
\hline 33 & $6-17-19-1$ & 0,381 & 0,120 & 0,008 & 89 & $6-11-2-1$ & 0,467 & 0,092 & 0,008 & 145 & $6-7-17-1$ & 0,449 & 0,206 & 0,010 & 201 & $6-19-1$ & 0,317 & 0,105 & 0,015 \\
\hline 34 & $6-19-2-1$ & 0,358 & 0,068 & 0,008 & 90 & $6-13-9-1$ & 0,445 & 0,086 & 0,008 & 146 & $6-9-11-1$ & 0,458 & 0,090 & 0,010 & 202 & $6-4-8-1$ & 0,438 & 0,100 & 0,015 \\
\hline 35 & $6-16-15-1$ & 0,393 & 0,088 & 0,008 & 91 & $6-17-13-1$ & 0,408 & 0,045 & 0,008 & 147 & $6-8-7-1$ & 0,441 & 0,062 & 0,010 & 203 & $6-19-3-1$ & 0,412 & 0,052 & 0,015 \\
\hline 36 & $6-16-17-1$ & 0,435 & 0,092 & 0,008 & 92 & $6-10-5-1$ & 0,432 & 0,056 & 0,008 & 148 & $6-7-19-1$ & 0,432 & 0,069 & 0,010 & 204 & $6-15-10-1$ & 0,443 & 0,090 & 0,016 \\
\hline 37 & $6-19-5-1$ & 0,386 & 0,083 & 0,008 & 93 & $6-18-11-1$ & 0,456 & 0,085 & 0,008 & 149 & $6-5-4-1$ & 0,459 & 0,151 & 0,010 & 205 & $6-11-10-1$ & 0,393 & 0,065 & 0,016 \\
\hline 38 & $6-20-16-1$ & 0,357 & 0,048 & 0,008 & 94 & $6-14-12-1$ & 0,428 & 0,067 & 0,008 & 150 & $6-10-2-1$ & 0,415 & 0,054 & 0,010 & 206 & $6-9-5-1$ & 0,477 & 0,112 & 0,016 \\
\hline 39 & $6-16-7-1$ & 0,419 & 0,056 & 0,008 & 95 & $6-17-15-1$ & 0,461 & 0,051 & 0,008 & 151 & $6-6-9-1$ & 0,454 & 0,091 & 0,010 & 207 & $6-6-17-1$ & 0,498 & 0,254 & 0,017 \\
\hline 40 & $6-19-20-1$ & 0,415 & 0,077 & 0,008 & 96 & $6-10-11-1$ & 0,433 & 0,052 & 0,008 & 152 & $6-7-13-1$ & 0,472 & 0,063 & 0,010 & 208 & $6-11-1$ & 0,377 & 0,154 & 0,017 \\
\hline 41 & $6-18-15-1$ & 0,396 & 0,114 & 0,008 & 97 & $6-10-16-1$ & 0,469 & 0,097 & 0,008 & 153 & $6-11-9-1$ & 0,373 & 0,053 & 0,010 & 209 & $6-14-1$ & 0,349 & 0,083 & 0,017 \\
\hline 42 & $6-17-14-1$ & 0,390 & 0,052 & 0,008 & 98 & $6-11-11-1$ & 0,414 & 0,053 & 0,008 & 154 & $6-9-1-1$ & 0,434 & 0,055 & 0,010 & 210 & $6-18-13-1$ & 0,400 & 0,044 & 0,018 \\
\hline 43 & $6-16-9-1$ & 0,386 & 0,047 & 0,008 & 99 & $6-9-10-1$ & 0,434 & 0,112 & 0,008 & 155 & $6-11-6-1$ & 0,410 & 0,053 & 0,010 & 211 & $6-5-1$ & 0,336 & 0,110 & 0,019 \\
\hline 44 & $6-18-12-1$ & 0,419 & 0,049 & 0,008 & 100 & $6-10-20-1$ & 0,498 & 0,056 & 0,008 & 156 & $6-7-15-1$ & 0,361 & 0,062 & 0,011 & 212 & $6-20-9-1$ & 0,421 & 0,050 & 0,022 \\
\hline 45 & $6-16-19-1$ & 0,406 & 0,077 & 0,008 & 101 & $6-12-6-1$ & 0,433 & 0,088 & 0,008 & 157 & $6-7-14-1$ & 0,482 & 0,105 & 0,011 & 213 & $6-6-1$ & 0,409 & 0,181 & 0,022 \\
\hline 46 & $6-11-18-1$ & 0,452 & 0,086 & 0,008 & 102 & $6-10-18-1$ & 0,456 & 0,101 & 0,009 & 158 & $6-9-6-1$ & 0,473 & 0,150 & 0,011 & 214 & $6-10-1$ & 0,387 & 0,122 & 0,022 \\
\hline 47 & $6-18-18-1$ & 0,431 & 0,128 & 0,008 & 103 & $6-12-13-1$ & 0,465 & 0,112 & 0,009 & 159 & $6-13-3-1$ & 0,394 & 0,054 & 0,011 & 215 & $6-3-1$ & 0,454 & 0,196 & 0,023 \\
\hline 48 & $6-17-12-1$ & 0,405 & 0,045 & 0,008 & 104 & $6-13-2-1$ & 0,459 & 0,072 & 0,009 & 160 & $6-5-6-1$ & 0,468 & 0,090 & 0,011 & 216 & $6-2-1$ & 0,360 & 0,257 & 0,023 \\
\hline 49 & $6-15-16-1$ & 0,430 & 0,056 & 0,008 & 105 & $6-16-12-1$ & 0,394 & 0,054 & 0,009 & 161 & $6-8-4-1$ & 0,448 & 0,057 & 0,011 & 217 & $6-9-4-1$ & 0,446 & 0,098 & 0,026 \\
\hline 50 & $6-16-14-1$ & 0,418 & 0,049 & 0,008 & 106 & $6-11-3-1$ & 0,475 & 0,126 & 0,009 & 162 & $6-4-5-1$ & 0,454 & 0,065 & 0,011 & 218 & $6-15-11-1$ & 0,388 & 0,153 & 0,027 \\
\hline
\end{tabular}


Table 3 Evaluation and selection of multiple network architectures (Continued)

\begin{tabular}{|c|c|c|c|c|c|c|c|c|c|c|c|c|c|c|c|c|c|c|c|}
\hline 51 & $6-16-4-1$ & 0,446 & 0,074 & 0,008 & 107 & $6-15-7-1$ & 0,429 & 0,094 & 0,009 & 163 & $6-5-10-1$ & 0,447 & 0,138 & 0,011 & 219 & $6-4-1$ & 0,477 & 0,187 & 0,027 \\
\hline 52 & $6-13-10-1$ & 0,424 & 0,049 & 0,008 & 108 & $6-10-19-1$ & 0,407 & 0,109 & 0,009 & 164 & $6-7-10-1$ & 0,462 & 0,118 & 0,011 & 220 & $6-19-10-1$ & 0,408 & 0,106 & 0,028 \\
\hline 53 & $6-18-20-1$ & 0,444 & 0,049 & 0,008 & 109 & $6-11-7-1$ & 0,440 & 0,044 & 0,009 & 165 & $6-8-12-1$ & 0,443 & 0,094 & 0,011 & 221 & $6-15-19-1$ & 0,445 & 0,094 & 0,029 \\
\hline 54 & $6-15-4-1$ & 0,449 & 0,045 & 0,008 & 110 & $6-14-4-1$ & 0,436 & 0,051 & 0,009 & 166 & $6-10-7-1$ & 0,412 & 0,110 & 0,011 & 222 & $6-15-17-1$ & 0,351 & 0,066 & 0,029 \\
\hline 55 & $6-14-10-1$ & 0,364 & 0,048 & 0,008 & 111 & $6-19-13-1$ & 0,377 & 0,091 & 0,009 & 167 & $6-6-12-1$ & 0,480 & 0,077 & 0,011 & 223 & $6-3-4-1$ & 0,479 & 0,161 & 0,030 \\
\hline 56 & $6-20-3-1$ & 0,356 & 0,047 & 0,008 & 112 & $6-11-14-1$ & 0,428 & 0,082 & 0,009 & 168 & $6-6-5-1$ & 0,408 & 0,139 & 0,011 & 224 & $6-6-13-1$ & 0,403 & 0,272 & 0,030 \\
\hline No. & ANN architecture & $\begin{array}{c}\text { MSE } \\
50 \\
\text { epochs }\end{array}$ & $\begin{array}{c}\text { MSE } \\
500 \\
\text { epochs }\end{array}$ & $\begin{array}{c}\text { MSE } \\
5000 \\
\text { epochs }\end{array}$ & No. & $\begin{array}{c}\text { ANN } \\
\text { architecture }\end{array}$ & $\begin{array}{c}\text { MSE } \\
50 \\
\text { epochs }\end{array}$ & $\begin{array}{c}\text { MSE } \\
500 \\
\text { epochs }\end{array}$ & $\begin{array}{c}\text { MSE } \\
5000 \\
\text { epochs }\end{array}$ & No. & $\begin{array}{c}\text { ANN } \\
\text { architecture }\end{array}$ & $\begin{array}{c}\text { MSE } \\
50 \\
\text { epochs }\end{array}$ & $\begin{array}{c}\text { MSE } \\
500 \\
\text { epochs }\end{array}$ & $\begin{array}{c}\text { MSE } \\
5000 \\
\text { epochs }\end{array}$ & No. & $\begin{array}{c}\text { ANN } \\
\text { architecture }\end{array}$ & $\begin{array}{c}\text { MSE } \\
50 \\
\text { epochs }\end{array}$ & $\begin{array}{c}\text { MSE } \\
500 \\
\text { epochs }\end{array}$ & $\begin{array}{c}\text { MSE } \\
5000 \\
\text { epochs }\end{array}$ \\
\hline 225 & $6-15-8-1$ & 0,357 & 0,051 & 0,031 & 281 & $6-7-8-1$ & 0,465 & 0,197 & 0,057 & 337 & $6-7-1$ & 0,421 & 0,111 & 0,065 & 393 & $6-5-3-1$ & 0,504 & 0,165 & 0,216 \\
\hline 226 & $6-10-15-1$ & 0,451 & 0,084 & 0,033 & 282 & $6-14-13-1$ & 0,398 & 0,056 & 0,057 & 338 & $6-3-9-1$ & 0,501 & 0,262 & 0,065 & 394 & $6-3-14-1$ & 0,500 & 0,263 & 0,218 \\
\hline 227 & $6-20-1-1$ & 0,369 & 0,043 & 0,034 & 283 & $6-8-2-1$ & 0,410 & 0,117 & 0,057 & 339 & $6-3-15-1$ & 0,489 & 0,185 & 0,065 & 395 & $6-2-6-1$ & 0,497 & 0,405 & 0,232 \\
\hline 228 & $6-17-1-1$ & 0,388 & 0,064 & 0,036 & 284 & $6-17-16-1$ & 0,405 & 0,094 & 0,057 & 340 & $6-18-17-1$ & 0,441 & 0,076 & 0,067 & 396 & $6-5-9-1$ & 0,495 & 0,107 & 0,232 \\
\hline 229 & $6-15-5-1$ & 0,401 & 0,057 & 0,036 & 285 & $6-19-6-1$ & 0,450 & 0,047 & 0,057 & 341 & $6-16-2-1$ & 0,441 & $0] 052$ & 0,068 & 397 & $6-2-18-1$ & 0,494 & 0,227 & 0,250 \\
\hline 230 & $6-7-16-1$ & 0,404 & 0,241 & 0,037 & 286 & $6-18-4-1$ & 0,387 & 0,053 & 0,057 & 342 & $6-9-18-1$ & 0,409 & 0,075 & 0,070 & 398 & $6-1-18-1$ & 0,501 & 0,495 & 0,251 \\
\hline 231 & $6-10-4-1$ & 0,456 & 0,114 & 0,037 & 287 & $6-12-14-1$ & 0,486 & 0,131 & 0,057 & 343 & $6-13-6-1$ & 0,478 & 0,063 & 0,071 & 399 & $6-1-16-1$ & 0,495 & 0,494 & 0,252 \\
\hline 232 & $6-16-20-1$ & 0,432 & 0,056 & 0,037 & 288 & $6-15-6-1$ & 0,471 & 0,047 & 0,057 & 344 & $6-17-7-1$ & 0,391 & 0,089 & 0,084 & 400 & $6-1-10-1$ & 0,499 & 0,489 & 0,254 \\
\hline 233 & $6-14-20-1$ & 0,389 & 0,050 & 0,038 & 289 & $6-8-13-1$ & 0,476 & 0,071 & 0,057 & 345 & $6-20-12-1$ & 0,410 & 0,037 & 0,085 & 401 & $6-1-8-1$ & 0,487 & 0,489 & 0,265 \\
\hline 234 & $6-20-20-1$ & 0,428 & 0,076 & 0,039 & 290 & $6-12-10-1$ & 0,362 & 0,053 & 0,057 & 346 & $6-9-1$ & 0,349 & 0,201 & 0,086 & 402 & $6-2-8-1$ & 0,462 & 0,235 & 0,283 \\
\hline 235 & $6-19-1-1$ & 0,417 & 0,132 & 0,042 & 291 & $6-14-16-1$ & 0,433 & 0,056 & 0,057 & 347 & $6-8-1-1$ & 0,434 & 0,097 & 0,090 & 403 & $6-2-13-1$ & 0,435 & 0,270 & 0,297 \\
\hline 236 & $6-6-14-1$ & 0,428 & 0,063 & 0,042 & 292 & $6-13-12-1$ & 0,448 & 0,057 & 0,057 & 348 & $6-4-7-1$ & 0,443 & 0,211 & 0,091 & 404 & $6-2-20-1$ & 0,419 & 0,326 & 0,298 \\
\hline 237 & $6-12-2-1$ & 0,399 & 0,059 & 0,043 & 293 & $6-12-1$ & 0,342 & 0,087 & 0,057 & 349 & $6-3-5-1$ & 0,479 & 0,247 & 0,094 & 405 & $6-1-5-1$ & 0,490 & 0,264 & 0,326 \\
\hline 238 & $6-20-4-1$ & 0,446 & 0,064 & 0,044 & 294 & $6-5-16-1$ & 0,477 & 0,062 & 0,057 & 350 & $6-16-1-1$ & 0,425 & 0,121 & 0,096 & 406 & $6-1-20-1$ & 0,497 & 0,440 & 0,328 \\
\hline 239 & $6-18-14-1$ & 0,352 & 0,038 & 0,044 & 295 & $6-13-1-1$ & 0,352 & 0,052 & 0,058 & 351 & $6-2-10-1$ & 0,399 & 0,318 & 0,097 & 407 & $6-3-7-1$ & 0,452 & 0,227 & 0,330 \\
\hline 240 & $6-18-1$ & 0,300 & 0,141 & 0,045 & 296 & $6-5-19-1$ & 0,456 & 0,075 & 0,058 & 352 & $6-2-3-1$ & 0,497 & 0,362 & 0,097 & 408 & $6-2-17-1$ & 0,495 & 0,347 & 0,344 \\
\hline 241 & $6-14-19-1$ & 0,410 & 0,049 & 0,045 & 297 & $6-16-18-1$ & 0,411 & 0,045 & 0,058 & 353 & $6-3-16-1$ & 0,473 & 0,207 & 0,098 & 409 & $6-1-11-1$ & 0,501 & 0,307 & 0,371 \\
\hline 242 & $6-14-7-1$ & 0,432 & 0,058 & 0,046 & 298 & $6-6-8-1$ & 0,443 & 0,109 & 0,058 & 354 & $6-4-12-1$ & 0,486 & 0,310 & 0,098 & 410 & $6-1-12-1$ & 0,495 & 0,249 & 0,371 \\
\hline
\end{tabular}


Table 3 Evaluation and selection of multiple network architectures (Continued)

\begin{tabular}{|c|c|c|c|c|c|c|c|c|c|c|c|c|c|c|c|c|c|c|c|}
\hline 243 & $6-18-9-1$ & 0,384 & 0,045 & 0,047 & 299 & $6-12-19-1$ & 0,473 & 0,057 & 0,058 & 355 & $6-17-11-1$ & 0,363 & 0,061 & 0,104 & 411 & $6-1-2-1$ & 0,495 & 0,435 & 0,382 \\
\hline 244 & $6-9-20-1$ & 0,377 & 0,050 & 0,048 & 300 & $6-7-7-1$ & 0,463 & 0,126 & 0,058 & 356 & $6-17-3-1$ & 0,388 & 0,048 & 0,106 & 412 & $6-1-13-1$ & 0,494 & 0,348 & 0,393 \\
\hline 245 & $6-10-3-1$ & 0,462 & 0,110 & 0,048 & 301 & $6-8-11-1$ & 0,410 & 0,102 & 0,058 & 357 & $6-14-1-1$ & 0,415 & 0,049 & 0,107 & 413 & $6-1-1$ & 0,435 & 0,486 & 0,408 \\
\hline 246 & $6-10-9-1$ & 0,502 & 0,052 & 0,048 & 302 & $6-12-17-1$ & 0,421 & 0,048 & 0,058 & 358 & $6-18-2-1$ & 0,353 & 0,079 & 0,107 & 414 & $6-1-1-1$ & 0,511 & 0,284 & 0,465 \\
\hline 247 & $6-20-18-1$ & 0,447 & 0,048 & 0,049 & 303 & $6-9-12-1$ & 0,391 & 0,116 & 0,059 & 359 & $6-7-18-1$ & 0,483 & 0,101 & 0,107 & 415 & $6-1-6-1$ & 0,488 & 0,301 & 0,476 \\
\hline 248 & $6-12-8-1$ & 0,413 & 0,048 & 0,049 & 304 & $6-11-12-1$ & 0,473 & 0,083 & 0,059 & 360 & $6-15-18-1$ & 0,443 & 0,065 & 0,107 & 416 & $6-1-15-1$ & 0,489 & 0,464 & 0,479 \\
\hline 249 & $6-8-17-1$ & 0,478 & 0,115 & 0,050 & 305 & $6-13-19-1$ & 0,390 & 0,064 & 0,059 & 361 & $6-3-3-1$ & 0,486 & 0,495 & 0,109 & 417 & $6-1-3-1$ & 0,478 & 0,381 & 0,488 \\
\hline 250 & $6-7-2-1$ & 0,470 & 0,062 & 0,050 & 306 & $6-10-13-1$ & 0,439 & 0,080 & 0,059 & 362 & $6-9-13-1$ & 0,439 & 0,090 & 0,110 & 418 & $6-1-4-1$ & 0,494 & 0,486 & 0,495 \\
\hline 251 & $6-14-6-1$ & 0,441 & 0,048 & 0,050 & 307 & $6-5-12-1$ & 0,477 & 0,279 & 0,059 & 363 & $6-2-12-1$ & 0,499 & 0,356 & 0,110 & 419 & $6-1-7-1$ & 0,484 & 0,427 & 0,496 \\
\hline 252 & $6-20-14-1$ & 0,403 & 0,043 & 0,051 & 308 & $6-10-8-1$ & 0,460 & 0,056 & 0,059 & 364 & $6-5-7-1$ & 0,428 & 0,163 & 0,110 & 420 & $6-1-19-1$ & 0,500 & 0,495 & 0,500 \\
\hline 253 & $6-13-14-1$ & 0,427 & 0,081 & 0,051 & 309 & $6-9-9-1$ & 0,417 & 0,075 & 0,059 & 365 & $6-4-15-1$ & 0,459 & 0,291 & 0,112 & 421 & $6-1-14-1$ & 0,500 & 0,495 & 0,501 \\
\hline 254 & $6-12-20-1$ & 0,440 & 0,050 & 0,051 & 310 & $6-3-11-1$ & 0,468 & 0,138 & 0,059 & 366 & $6-2-15-1$ & 0,468 & 0,334 & 0,113 & & & & & \\
\hline 255 & $6-14-5-1$ & 0,403 & 0,045 & 0,052 & 311 & $6-7-5-1$ & 0,455 & 0,071 & 0,060 & 367 & $6-6-10-1$ & 0,445 & 0,070 & 0,114 & & & & & \\
\hline 256 & $6-8-6-1$ & 0,449 & 0,053 & 0,052 & 312 & $6-6-2-1$ & 0,496 & 0,087 & 0,060 & 368 & $6-5-13-1$ & 0,418 & 0,353 & 0,114 & & & & & \\
\hline 257 & $6-14-11-1$ & 0,422 & 0,072 & 0,053 & 313 & $6-12-9-1$ & 0,406 & 0,125 & 0,060 & 369 & $6-5-18-1$ & 0,449 & 0,331 & 0,115 & & & & & \\
\hline 258 & $6-13-15-1$ & 0,430 & 0,071 & 0,053 & 314 & $6-4-6-1$ & 0,406 & 0,269 & 0,060 & 370 & $6-4-19-1$ & 0,482 & 0,183 & 0,116 & & & & & \\
\hline 259 & $6-7-9-1$ & 0,453 & 0,106 & 0,053 & 315 & $6-5-5-1$ & 0,482 & 0,068 & 0,060 & 371 & $6-5-2-1$ & 0,476 & 0,216 & 0,118 & & & & & \\
\hline 260 & $6-17-10-1$ & 0,464 & 0,047 & 0,053 & 316 & $6-6-11-1$ & 0,487 & 0,075 & 0,060 & 372 & $6-2-14-1$ & 0,480 & 0,279 & 0,119 & & & & & \\
\hline 261 & $6-12-18-1$ & 0,439 & 0,051 & 0,053 & 317 & $6-6-19-1$ & 0,361 & $0] 121$ & 0,061 & 373 & $6-20-15-1$ & 0,414 & 0,048 & 0,128 & & & & & \\
\hline 262 & $6-11-15-1$ & 0,367 & 0,096 & 0,053 & 318 & $6-4-2-1$ & 0,465 & 0,190 & 0,061 & 374 & $6-4-14-1$ & 0,439 & 0,074 & 0,130 & & & & & \\
\hline 263 & $6-17-5-1$ & 0,464 & 0,132 & 0,053 & 319 & $6-5-1-1$ & 0,410 & 0,131 & 0,061 & 375 & $6-3-13-1$ & 0,492 & 0,277 & 0,134 & & & & & \\
\hline 264 & $6-11-13-1$ & 0,491 & 0,154 & 0,054 & 320 & $6-7-6-1$ & 0,496 & 0,113 & 0,061 & 376 & $6-4-18-1$ & 0,462 & 0,136 & 0,135 & & & & & \\
\hline 265 & $6-7-11-1$ & 0,458 & 0,062 & 0,054 & 321 & $6-9-17-1$ & 0,432 & 0,146 & 0,061 & 377 & $6-2-9-1$ & 0,469 & 0,433 & 0,136 & & & & & \\
\hline 266 & $6-19-16-1$ & 0,424 & 0,165 & 0,055 & 322 & $6-3-19-1$ & 0,488 & 0,285 & 0,061 & 378 & $6-1-17-1$ & 0,456 & 0,382 & 0,141 & & & & & \\
\hline 267 & $6-16-1$ & 0,327 & 0,064 & 0,055 & 323 & $6-2-19-1$ & 0,486 & 0,279 & 0,061 & 379 & $6-4-11-1$ & 0,389 & 0,150 & 0,141 & & & & & \\
\hline 268 & $6-20-5-1$ & 0,427 & 0,076 & 0,055 & 324 & $6-3-10-1$ & 0,495 & 0,250 & 0,061 & 380 & $6-1$ & 0,300 & 0,218 & 0,143 & & & & & \\
\hline
\end{tabular}


Table 3 Evaluation and selection of multiple network architectures (Continued)

\begin{tabular}{|c|c|c|c|c|c|c|c|c|c|c|c|c|c|c|}
\hline 269 & $6-11-8-1$ & 0,456 & 0,054 & 0,055 & 325 & $6-4-9-1$ & 0,436 & 0,222 & 0,062 & 381 & $6-3-18-1$ & 0,392 & 0,228 & 0,143 \\
\hline 270 & $6-14-15-1$ & 0,422 & 0,060 & 0,055 & 326 & $6-9-19-1$ & 0,464 & 0,098 & 0,062 & 382 & $6-4-16-1$ & 0,461 & 0,081 & 0,143 \\
\hline 271 & $6-13-7-1$ & 0,439 & 0,049 & 0,055 & 327 & $6-7-12-1$ & 0,418 & 0,219 & 0,062 & 383 & $6-2-2-1$ & 0,450 & 0,451 & 0,151 \\
\hline 272 & $6-17-1$ & 0,350 & 0,079 & 0,055 & 328 & $6-2-1-1$ & 0,503 & 0,495 & 0,062 & 384 & $6-2-5-1$ & 0,488 & 0,354 & 0,152 \\
\hline 273 & $6-12-15-1$ & 0,470 & 0,048 & 0,055 & 329 & $6-8-1$ & 0,411 & 0,119 & 0,062 & 385 & $6-11-5-1$ & 0,483 & 0,099 & 0,154 \\
\hline 274 & $6-19-7-1$ & 0,420 & 0,049 & 0,056 & 330 & $6-4-3-1$ & 0,504 & 0,288 & 0,062 & 386 & $6-2-7-1$ & 0,495 & 0,365 & 0,168 \\
\hline 275 & $6-15-9-1$ & 0,474 & 0,058 & 0,056 & 331 & $6-14-17-1$ & 0,377 & 0,092 & 0,063 & 387 & $6-3-17-1$ & 0,395 & 0,306 & 0,170 \\
\hline 276 & $6-11-20-1$ & 0,369 & 0,063 & 0,056 & 332 & $6-12-3-1$ & 0,451 & 0,063 & 0,063 & 388 & $6-3-1-1$ & 0,476 & 0,203 & 0,177 \\
\hline 277 & $6-19-12-1$ & 0,414 & 0,043 & 0,056 & 333 & $6-13-1$ & 0,298 & 0,128 & 0,063 & 389 & $6-2-11-1$ & 0,493 & 0,319 & 0,181 \\
\hline 278 & $6-19-17-1$ & 0,404 & 0,093 & 0,056 & 334 & $6-13-13-1$ & 0,479 & 0,048 & 0,064 & 390 & $6-1-9-1$ & 0,495 & 0,485 & 0,209 \\
\hline 279 & $6-20-2-1$ & 0,425 & 0,080 & 0,056 & 335 & $6-7-4-1$ & 0,453 & 0,134 & 0,064 & 391 & $6-2-4-1$ & 0,496 & 0,426 & 0,212 \\
\hline 280 & $6-19-8-1$ & 0,420 & 0,065 & 0,056 & 336 & $6-2-16-1$ & 0,485 & 0,284 & 0,064 & 392 & $6-6-6-1$ & 0,442 & 0,194 & 0,214 \\
\hline
\end{tabular}

The models with the least MSE are marked in bold. 
representation of the best architecture of artificial neural network for our problem is shown in Figure 1. The number of neurons in the input layer was 6 and it was equal to the number of ACTB and GAPDH expression measurements. The ideal outputs were set at 1 for the positive history of IS in the family and at 0 for absence of IS in the anamnesis. The number of hidden nodes was obtained by trial and error method. We trained 421 neural networks models with different number of hidden nodes using the backpropagation algorithm (activation function: binary sigmoidal function, learning rate: 0,1 ; momentum rate: 0,01 ; epochs: 50,500 and 5000) and the training set. The backpropagation teaching method was chosen because it is the most common method of training multilayered feed-forward neural networks [30]. Initially, 50 training epochs were considered but it did not yield a satisfactory result (Table 3). The mean square error (MSE) was high. This MSE was minimized by increasing the epochs from 50 to 500 and finally from 500 to 5000 [Table 3]. Thereafter, we selected 3 neural networks with the least mean square error (MSE) for training set. To test the classification ability of the ANN approach, we used the selected neural models and test set of data. The ANN model with the best classification accuracy for Idiopathic Scoliosis in the anamnesis with expression measurement of ACTB and GAPDH was chosen as the best.

\section{Results}

The data have been analyzed using NeuronDotNet computer library [32]. Training an ANN is the process of setting the best weights on the inputs of each of the nodes. The goal is to use the training set to produce weights where the output of the network is as close to the desired output as possible for as many of the examples in the training set as possible [30]. Table 3 shows the MSE for all 421 trained artificial neural models. A satisfactory MSE was yielded for ANNs with:

- 18 nodes in the first hidden layer and 16 nodes in the second hidden layer

- 19 nodes in the first hidden layer and 19 nodes in the second hidden layer

- 18 nodes in the first hidden layer and 10 nodes in the second hidden layer

Figure 2 presents the MSE for ANN model based on 6-18-16-1 architecture and the training set.

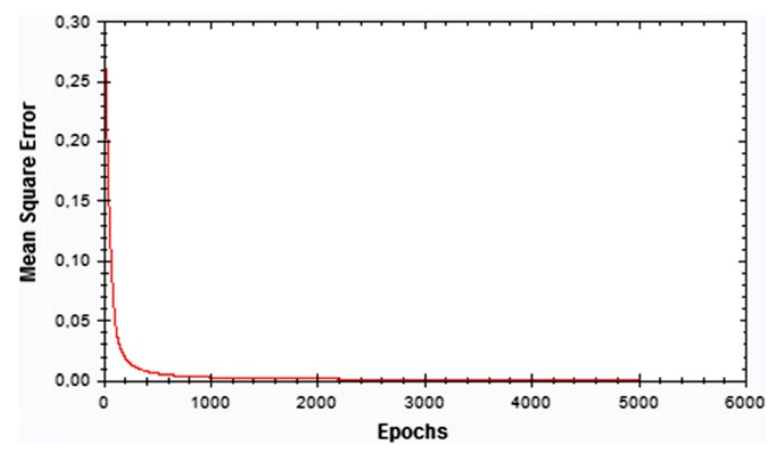

Figure 2 Plot of total error in training ANN based on 6-18-16-1 architecture. Training of the feedforward backpropagation neural network as measured by the square error of the difference between the actual and predicted variable. 
Table 2 lists classification results on the test set of ANN modelling for presence and absence of Idiopathic Scoliosis in the anamnesis. It proves how well the artificial neural network will perform on new data. The comparison of developed models showed, that the most satisfactory classification accuracy was achieved for ANN model with 18 nodes in the first hidden layer and 16 nodes in the second hidden layer. The classification accuracy for Idiopathic Scoliosis in the anamnesis with expression measurement of ACTB and GAPDH with use of ANN based on 6-18-16-1 architecture was 8 of 9 (88\%). Only in one case (ID 27 in test set), the prediction was ambiguous.

\section{Conclusions}

The results of this study confirm the potential benefits of the artificial neural network application for clinical research and point at human housekeeping genes as a potential target for future molecular investigations on idiopathic scoliosis etiopathogenesis. The analysis indicates the relationship between level of expression of ACTB, GAPDH and familial Idiopathic Scoliosis.

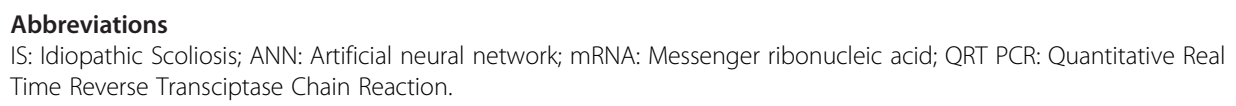
measurements and statistical analysis and drafted the manuscript. TW carried out analysis based on artificial neural network and participated in the design of the study. MT has supported us with her experience and knowledge concerning advanced data analysis: knowledge discovery from data, data mining, artificial intelligence and machine learning, and together with DZ and UM has been involved in the design of the study and interpretation of the data and helped to draft the manuscript. All authors read and approved the final manuscript.

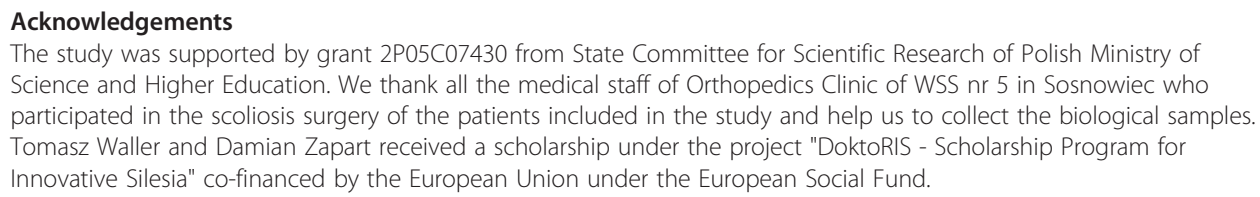
Science and Higher Education. We thank all the medical staff of Orthopedics Clinic of WSS nr 5 in Sosnowiec who participated in the scoliosis surgery of the patients included in the study and help us to collect the biological samples. Tomasz Waller and Damian Zapart received a scholarship under the project "DoktoRIS - Scholarship Program for Innovative Silesia" co-financed by the European Union under the European Social Fund.

\section{Author details}

1 Institute of Computer Science, Division of Biomedical Computer Systems, University of Silesia, Katowice, Poland. ${ }^{2}$ Department and Clinic of Orthopaedics Medical University of Silesia, Sosnowiec, Poland. ${ }^{3}$ Department of Molecular Biology, Medical University of Silesia, Katowice, Poland. Institute of Computer Science, Division of Information Systems, University of Silesia, Katowice, Poland.

Received: 9 August 2012 Accepted: 4 December 2012

Published: 4 January 2013

References

1. Cheng JC, Tang NLS, Yeung H, Miller N: Genetic association of complex traits. Clin Ortop and Rel Res 2007, 462:36-44.

2. Grauers A, Rahman I, Gerdhem P: Heritability of scoliosis. Eur Spine J 2011, ahead of print.

3. Cowell HR, Hall JN, MacEwen GD: Genetic aspects of idiopathic scoliosis. Clin Orthop 1972, 86:121-131.

4. Miller NH: Genetics of familial idiopathic scoliosis. Clin Orthop Rel Res 2002, 401:60-64.

5. Miller NH: Genetics of familial idiopathic scoliosis. Clin Orthop and Rel Res 2007, 462:6-10.

6. Harrington PR: The etiology of idiopathic scoliosis. Clin Orthop 1977, 126:43-46.

7. Riseborough EJ, Wynne-Davies R: A genetic survey of of idiopathic scoliosis in Boston, Massachusetts. J Bone Joint Surg 1973, 55A:974-982.

8. Wynne-Davies R: Familial (idiopathic) scoliosis: a family survey. J Bone Joint Surg 1968, 50B:24-30.

9. Inoue M, Minami S, Kitahara H, Otsuka Y, Nakata Y, Takso M, Moria H: Idiopathic scoliosis In twins studied by DNA fingerprinting. J Bone and Joint Surg 1998, 80-B(2):212-217.

10. Kesling KL, Reinker KA: Scoliosis in twins. A metaanalysis of the literature and report of six cases. Spine 1997, 22:2009-2015 
11. Ogilvie JW, Braun J, Argyle VA, Nelson L, Meade M, Ward K: The search for idiopathic scoliosis genes. Spine 2006, 31(6):679-681.

12. Glare EM, Divjak M, Bailey MJ, Walters EH: $\beta$-actin and GAPDH housekeeping gene expression in asthmatic airways is variable and not suitable for normalizing mRNA levels. Thorax 2002, 57:765-770.

13. Cheng W, Chang C, Chen C, Tsai M, Shu W, Li C: Identification of reference genes across physiological states for qRT-PCR through microarray meta-analysis. PLoS One 2011, 6(2):e17347. http://www.plosone.org/article/info \%3Adoi\%2F10.1371\%2Fjournal.pone.0017347.

14. Shaw GT, Shih ES, Chen C, Hwang M: Preservation of ranking order in the expression of human housekeeping genes. PLoS One 2011, 6(12):e29314. http://www.plosone.org/article/info\%3Adoi\%2F10.1371\%2Fjournal. pone.0029314

15. Lenke LG, Betz RR, Harms J, Bridwell KH, Clements DH, Lowe TG, Blanke K: Adolescent idiopathic scoliosis. A new classification to determine extent of spinal arthrodesis. J Bone Joint Surg 2001, 83-A(8):1169-1181.

16. Aaro S, Dahlborn M: Estimation of vertebral rotation and the spinal and rib cage deformity in scoliosis by computer tomography. Spine 1981, 6:460-467.

17. Enders CK: Applied Missing Data Analysis. 72 Spring Street, New York: The Guilford Press; 2010. chapter 1.3: Missing Data Patterns,

18. Wang J: Data Mining: Opportunities and Challenges. Idea Group Publishing. 2003. chap 7: The impact of Missing Data on Data Mining.

19. Kantardzic M: Data Mining: Concepts, Models, Methods, and Algorithms. Secondth edition. Hoboken, NJ, USA: IEEE Press, John Wiley \& Sons, Inc; 2011. chapter 2.4 Missing Data.

20. Microsoft MSDN Analysis Services - Data Mining Documentation. http://msdn.microsoft.com/en-us/library/cc280406. aspx.

21. Han J, Kamber M: Data Mining: Concepts and Techniques: Concepts and Techniques. 3dth edition. The Morgan Kaufmann Series In Data Management Systems: Morgan Kaufmann Publishers; 2012. chapter 3.2.1 Missing Values.

22. Wang $\mathrm{H}$, Wang $\mathrm{S}$ : Discovering patterns of missing data in survey databases: an application of rough sets. Expert Syst Appl 2009, 36(3):6256-6260.

23. Tkacz M: Processing an Incomplete Data Using Artificial Neural Networks. Ostrava: International Workshop Control and Information Technology; 1999.

24. Tkacz M: Processing an Incomplete and Random Data Using Artificial Neural Networks. Ostrava: International Workshop Control and Information Technology; 2001.

25. Tkacz M: Geoenvironmental Modelling with Artificial Intelligence Methods in Case of Hybrid Geothermal System. Sosnowiec, Poland: PhD Thesis, in Polish: Modelowanie warunków geośrodowiskowych przy użyciu metod sztucznej inteligencji na przykładzie hybrydowego systemu płytkiej geotermiki), University of Silesia; 2004.

26. Tkacz M: Artificial Neural Networks in Incomplete Data Sets Processing. In Intelligent Information Processing and Web Mining. Advances in Soft Computing. Edited by Kłopotek MA, Wierzchon S, Trojanowski K. Berlin Heidelberg: Springer-Verlag; 2005.

27. Kłopotek MA, Wierzchon S, Trojanowski K, Tkacz M: Artifcial Neural Networks Resistance To Incomplete Data. In Intelligent Information Processing and Web Mining. Advances in Soft Computing. Berlin Heidelberg: Springer-Verlag; 2006.

28. Kan T, Shimada Y, Sato F, Ito T, Kondo K, Watanabe G, Maeda M, Yamasaki S, Meltzer SJ, Imamura M: Prediction of Lymph Node Metastasis with Use of Artificial Neural Networks Based on Gene Expression Profiles in Esophageal Squamous Cell Carcinoma. Ann Surg Oncol 2004, 11(12):1070-1078.

29. Peterson LE, Mustafa O, Halime E, Andrew A, Lori G, Collen CN, Michael I: Artificial Neural Network Analysis of DNA Microarray-based Prostate Cancer Recurrence, Proceedings of the 2005 IEEE Symposium on Computational Intelligence in Bioinformatics and Computational Biology.

30. Shanthi D, Sahoo G, Saravanan N: Designing an Artificial Neural Network Model for the Prediction of Thromboembolic Stroke. International Journals of Biometric and Bioinformatics (IJBB) 2009, 3(1):10-18.

31. van der Smagt P, Krose B: An Introduction to Neural Networks. Amsterdam: The University of Amsterdam; 1996.

32. NeuronDotNet. 2012. http://sourceforge.net/projects/neurondotnet/.

doi:10.1186/1475-925X-12-1

Cite this article as: Waller et al: Familial or Sporadic Idiopathic Scoliosis - classification based on artificial neural network and GAPDH and ACTB transcription profile. BioMedical Engineering OnLine 2013 12:1.

\section{Submit your next manuscript to BioMed Central and take full advantage of:}

- Convenient online submission

- Thorough peer review

- No space constraints or color figure charges

- Immediate publication on acceptance

- Inclusion in PubMed, CAS, Scopus and Google Scholar

- Research which is freely available for redistribution

Submit your manuscript at www.biomedcentral.com/submit 